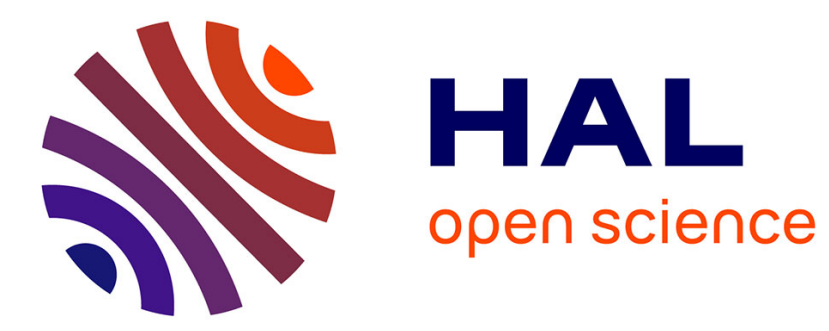

\title{
Some results on the controllability of planar variational inequalities
}

Bernard Brogliato

\section{To cite this version:}

Bernard Brogliato. Some results on the controllability of planar variational inequalities. RR-4788, INRIA. 2003. inria-00071798

\section{HAL Id: inria-00071798 \\ https://hal.inria.fr/inria-00071798}

Submitted on 23 May 2006

HAL is a multi-disciplinary open access archive for the deposit and dissemination of scientific research documents, whether they are published or not. The documents may come from teaching and research institutions in France or abroad, or from public or private research centers.
L'archive ouverte pluridisciplinaire HAL, est destinée au dépôt et à la diffusion de documents scientifiques de niveau recherche, publiés ou non, émanant des établissements d'enseignement et de recherche français ou étrangers, des laboratoires publics ou privés. 
INSTITUT NATIONAL DE RECHERCHE EN INFORMATIQUE ET EN AUTOMATIQUE

Some results on the controllability of planar variational inequalities

Bernard Brogliato

$\mathbf{N}^{\circ} 4788$

April 2003

THÈME 4 



\title{
Some results on the controllability of planar variational inequalities
}

\author{
Bernard Brogliato * \\ Thème 4 - Simulation et optimisation \\ de systèmes complexes \\ Projet Bipop \\ Rapport de recherche $n^{\circ} 4788$ - April 2003 - 13 pages
}

\begin{abstract}
This note deals with the controllability of a class of planar complementarity dynamical systems, which can also be viewed as planar variational inequalities. It is shown that the complementarity conditions influence a lot the controllability of the system.
\end{abstract}

Key-words: variational inequalities, complementarity systems, projected dynamics, unilateral dynamics, controllability.

* INRIA Rhône-Alpes, ZIRST Montbonnot, 655 avenue de l'Europe, 38334 Saint-Ismier, France, Bernard.Brogliato@inrialpes.fr 


\section{Résultats préliminaires sur la commandabilité des inéquations variationnelles en $2 \mathrm{D}$}

Résumé : Cette note concerne la commandabilité d'une classe de systèmes de complémentarité, qui peuvent être vus comme des inéquations variationnelles de dimension 2 . On montre que les conditions de complémentarité influencent largement la commandabilité de ces systèmes.

Mots-clés : Inéquations variationnelles, systèmes de complémentarité, dynamique projetée, dynamique unilatérale, commandabilité. 


\section{Introduction}

Hybrid dynamical systems constitute a very large class of systems [2]. It is consequently necessary to focus on specific subclasses to make their study possible, see e.g. [14] [15] [16] for controllability issues in piecewise-linear systems. An interesting subclass is made of so-called complementarity systems [13] [1]. Similarly to the fact that the stability of unilaterally constrained systems can significantly differ from that of their unconstrained counterpart [3] [4], it will be shown that their controllability properties can differ a lot as well. This reinforces the fact that such nonsmooth dynamical systems deserve full attention and are not a mere extension of unconstrained or bilaterally constrained systems. In this note we will restrict ourselves to a narrow class of complementarity systems, that we call planar evolution variational inequalities. These systems are also sometimes called projected dynamical systems [3] [6] and are used to model the dynamics of oligopolistic markets, spatial price equilibrium, elastic demand traffic equilibirum [3]. As illustrated at the end of the note, they can also model some electrical circuits with ideal diodes. In this note it is shown that the controllability of such systems, with piecewise continuous inputs, depend a lot on the convex set that within which the state is constrained to evolve.

\section{Planar evolution variational inequalities}

The linear complementarity systems (LCS) [1] we are dealing with in this study, possess the following dynamics

$$
\left\{\begin{array}{l}
\dot{z}_{1}=z_{2}+C_{1}^{T} \lambda \\
\dot{z}_{2}=u+C_{2}^{T} \lambda \\
0 \leq \lambda \perp C z+d \geq 0
\end{array}\right.
$$

where $C=\left(C_{1} C_{2}\right) \in \mathbb{R}^{m \times 2}, C_{1} \in \mathbb{R}^{m}$ and $C_{2} \in \mathbb{R}^{m}$ are the two columns of $C, d \in \mathbb{R}^{2}$, $\lambda \in \mathbb{R}^{m}$. The non-negativity is understood componentwise. The LCS in (1) is equivalent to the linear evolution variational inequality (VI)

$$
\left\{\begin{array}{l}
\langle\dot{z}+A z+B u, v-z\rangle \geq 0, \forall v \in K \\
z(t) \in K, \forall t \geq 0
\end{array}\right.
$$

where $z=\left(z_{1}, z_{2}\right)^{T} \in \mathbb{R}^{2}, A=\left(\begin{array}{cc}0 & 1 \\ 0 & 0\end{array}\right), B=\left(\begin{array}{c}0 \\ 1\end{array}\right), K=\left\{\left(z_{1}, z_{2}\right) \in \mathbb{R}^{2} \mid C_{1} z_{1}+C_{2} z_{2}+\right.$ $d \geq 0\}$.

It is noteworthy that, seen from an LCS point of view, the controlled dynamics in (1) is rather a narrow class. However the VI formalism in (2) shows that it is not so restrictive from an application point of view, since VI are widely used in some domains of science 
(see [3] for market and finance applications). VI can also represent some electrical circuits with ideal diodes [4]. The LCS in (1) is a particular gradient complementarity system [7], which is in turn equivalent to so-called projected dynamical systems [3] [5] [6]. So our study could be that of controllability of particular planar projected dynamical systems. There certainly remains a big gap between this work, and obtaining similar results for general projected dynamical systems (to say nothing for general LCS). It is worthy to remark that only the state space transformations $x=L z$ with $L L^{T}=I_{2}$, where $I_{2}$ is the $2 \times 2$ identity, preserve the variational inequality formalism in (2) [4]. Consequently the results apply to a restricted class of planar evolution VI only. Since the studies on controllability of this type of dynamical systems are rare, this paper nevertheless has some interest.

Remark 1 A more general class of systems consists of choosing $A=\left(\begin{array}{ll}a & 1 \\ 0 & 0\end{array}\right)$ for some real a (the second row in $A$ can easily be set to $(0,0)$ by feedback). Then applying a backstepping procedure [9] and a pre-feedback input $u=\bar{u}-a^{2} z_{1}-a z_{2}$, one can put the system under the form

$$
\left\{\begin{array}{l}
\langle\dot{\tilde{z}}+A \tilde{z}+B \bar{u}, w-\tilde{z}\rangle \geq 0, \forall w \in \tilde{K}\left(z_{1}\right) \\
\tilde{z}(t) \in \tilde{K}\left(z_{1}\right), \forall t \geq 0
\end{array}\right.
$$

where $\tilde{z}=\left(z_{1}, \tilde{z}_{2}\right)^{T}, \tilde{z}_{2}=z_{2}-z_{2 d}, z_{2 d}=-a z_{1}, \tilde{K}\left(z_{1}\right)=\{w \mid w=v-\tilde{z}, v \in K\}=$ $\left\{w \mid C w+d+C\left(\begin{array}{c}0 \\ a z_{1}\end{array}\right) \geq 0\right\}$. The system in (3) is therefore a VI with state dependent convex set $\tilde{K}\left(z_{1}\right)$ : the set $\tilde{K}$ moves as the state evolves. The well-posedness studies for such VI seem for the moment limited to Moreau's sweeping process [10, §3.3], i.e. with $A=0$. The obtained system is also called a parabolic quasi-variational inequality. We post-pone the study of such dynamical controlled systems to a future time.

The results in [4] and [8] assure that continuous solutions of (2) with locally differentiable derivative exist and are unique on $[0,+\infty)$, for all continuous and locally differentiable inputs $u(\cdot)$.

Definition 1 The system in (1) (equivalently in (2)) is said to be $K$-controllable, if any state $z_{f} \in K$ can be reached from any state $z_{i} \in K$, in a finite or infinite time $T$, and with an admissible input $u(\cdot)$.

Admissibility of the input means that the well-posedness conditions are respected. We do not make the difference between finite and infinite $T$ to simplify the presentation.

The objective of this work is to prove that, under some restrictions on the convex set $K$, the state $x(\cdot)$ can be steered inside $K$ (including the boundary $\partial K$ ) from any $x_{0} \in K$ to any $x_{1} \in K$. To begin with and to motivate the study, let us remark that in case $m=1$ and $K=\left\{z \mid z_{2} \geq-c, c<0\right\}$, then surely the system is not $K$-controllable. Indeed $z_{1}$ can only move from the left to the right in the phase plane, since $\dot{z}_{1}=z_{2} \geq-c>0$. 
This controlled VI is accessible [11] with reachable subspaces from $\left(z_{1}(0), z_{2}(0)\right)$ equal to $\left\{\left(z_{1}, z_{2}\right) \mid z_{1} \geq z_{1}(0), z_{2} \geq-c\right\}$, but not $K$-controllable.

Remark 2 It is not clear how this simple uncontrollable case, could be extended to higher dimensions.

Let us note that adding some "imaginary" state re-initialization rules on $\partial K$ such that $K$-controllability holds, is not envisaged here since the dynamical systems in (1) or (2) are the topic of the study. However motivated by this simple example of non-controllability, one guesses that a crucial step in the study will be to prove whether or not one is able to move on $\partial K$ in order to reach some states which are otherwise unreachable. Due to the complementarity conditions (third line in (1)) which imply that the vector field is modified when $\partial K$ is attained, this will under certain conditions be possible.

\section{Main result}

The following assumption is made and supposed to hold in the sequel:

Assumption 1 The set $K$ has a positive measure in $\mathbb{R}^{2}$.

It is easy to construct $C$ and $d$ such that indeed $K=\emptyset$ or it has zero measure.

Let us denote the faces of the convex set $K$ as $D^{i}$, such that $D^{i} \subseteq\left\{z \mid a_{i} z_{1}+b_{i} z_{2}+d_{i}=0\right\}$. In other words the faces are segments (possibly unbounded, like in the case $K$ is a cone, or if $K$ is defined as a half-space), and the segments can be extended to straight lines whose equations in the plane are $a_{i} z_{1}+b_{i} z_{2}+d_{i}=0,1 \leq i \leq m$. Thus $C_{1}=\left(a_{1}, \ldots, a_{m}\right)^{T}$, and $C_{2}=\left(b_{1}, \ldots, b_{m}\right)^{T}, d=\left(d_{1}, \ldots, d_{m}\right)^{T}$. Let us place ourselves in the phase plane of the system, with the two axis $\left(0, z_{1}\right)$ and $\left(0, z_{2}\right)$. Then the following is true

Lemma 1 The system in (1) (equivalently in (2)) is $K$-controllable if and only if there is no face of $K$ such that:

- there is a portion of $D^{i}$ with finite negative slope on the right (resp. left) of the point $D^{i} \cap\left\{z \mid z_{1}=0\right\}$, when $K$ is below (resp. above) $D^{i}$.

- $D^{i}$ is vertical and above (resp. below) $\left\{z \mid z_{1}=0\right\}$ if $K$ is on the right (resp. left) of $D^{i}$.

- $D^{i}$ is horizontal and in the half-space $\left\{z \mid z_{2}<0\right\}$ (resp. $\left\{z \mid z_{2}>0\right\}$ ) if $K$ is below (resp. above) $D^{i}$.

- $D^{i}=\left\{z \mid z_{2}=0\right\}$. 
An illustration of these various cases is provided in figure 1 . The system is not $K_{1}$-controllable, because the only way to attain a point on the left of the vertical line $(l)$ from a point on the right of $(l)$, is to follow the boundary of $K$. However once the point $A$ has been reached, it is impossible to move on $\partial K_{1}$ towards $A^{\prime}$. The system can be steered on the line $A A^{\prime}$ only in the direction of $B$. Therefore all points in $K_{1}$ which are situated on the left of $(l)$, cannot be attained from points in $K_{1}$ on the right of $(l)$. The system is $K_{2}$-controllable, however adding the dashed portion to $K_{2}$, one obtains $K_{4}$ and the system is not $K_{4}$-controllable. Indeed the points in $K_{4}$ on the left of $(l)$, can be reached only by following $\partial K_{4}$ along $C D$. It is possible to move along the face $E C$ from $E$ to $C$, but $C$ can be attained only asymptotically, and it cannot be crossed. Thus points on $C D$ cannot be reached from the right of $(l)$, and the portion of $K_{4}$ that is on the left of $(l)$ is unreachable from points on the right of $(l)$. The system is $K_{3}$-controllable.

These various results are a consequence of the complementarity conditions in (1), which preclude the use of any control input $u(\cdot)$ on $\partial K: u(\cdot)$ has to assure that the trajectory remains on $\partial K$.

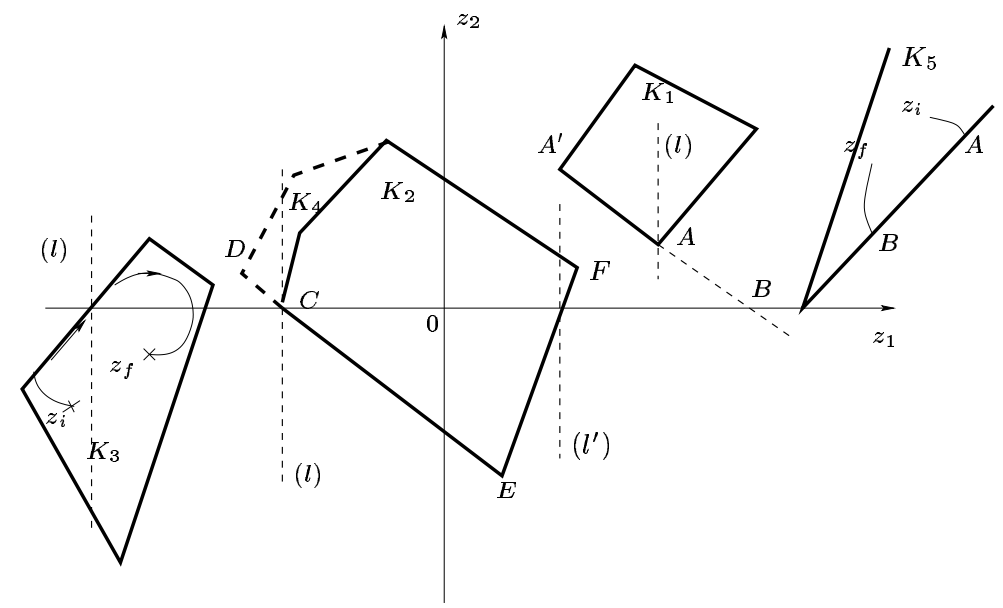

Figure 1: Examples of $K$-controllable and $K$-uncontrollable systems.

Before proving lemma 1, let us state intermediate results which characterize the motion on the boundary $\partial \Phi$.

Proposition 1 Let us consider the system in (1) or (2), with $C_{1}=a \in \mathbb{R}, C_{2}=b \in \mathbb{R}$, $d=c \in \mathbb{R}$. Let us define the coordinate change $\left\{\begin{array}{l}x_{1}=b z_{1}-a z_{2}+\frac{b c}{a} \\ x_{2}=a z_{1}+b z_{2}+c\end{array}\right.$. We denote as $P$ the intersection between the line $a z_{1}+b z_{2}+c=0$ and the $z_{1}$-axis, i.e. $P$ is the origin of the new frame $\left(x_{1}, x_{2}\right)$ and the constraint boundary is $\left\{x \mid x_{2}=0\right\}$. Then 


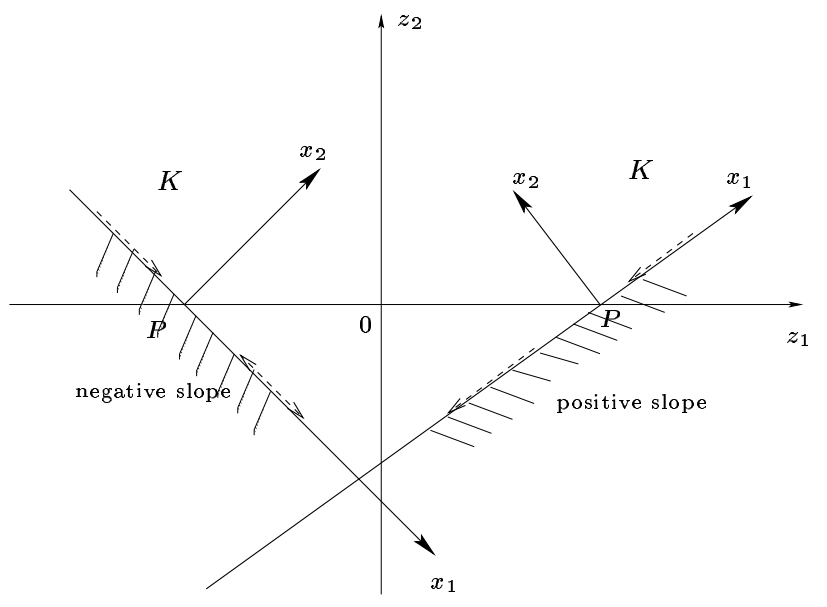

Figure 2: The new coordinate frame.

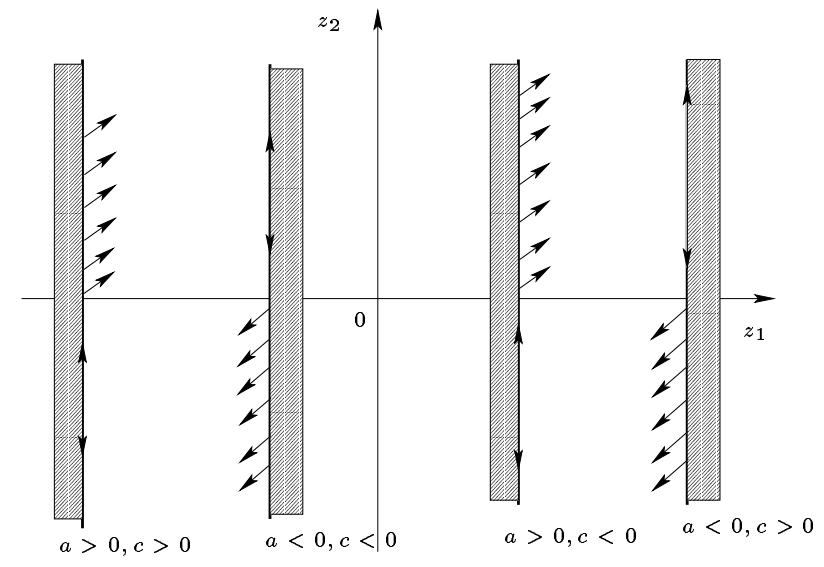

Figure 3: Trajectories on $\partial \Phi$ (vertical faces).

$\mathrm{RR} \mathrm{n}^{\circ} 4788$ 
- (positive slope) If $-\frac{a}{b}>0, b \neq 0$, any point $x_{1 f}$ on the constraint can be attained from any point $x_{1 i} \geq x_{1 f}$.

- (negative slope) If $-\frac{a}{b}<0, b \neq 0$, then any point $x_{1 f}$ on the constraint can be attained from any point $x_{1 i} \leq x_{1 f}$, and any point $x_{1 f}$ on the constraint can be attained from any point $x_{1 i} \geq x_{1 f}$ only on the axis $x_{1} \in[P,+\infty)$. Moreover the point $P$ can be attained from any $x_{1 i}>0$ only asymptotically.

If $a=0$ then the boundary is a horizontal line $z_{2}=-\frac{c}{b}$ and

- if $(b>0$ and $c<0)$ or $(b<0$ and $c>0)$, trajectories move from the left to the right,

- if $(b>0$ and $c>0)$ or $(b<0$ and $c<0)$, trajectories move from the right to the left,

- if $c=0$ then the system remains stuck on $\partial K$ at the contacting point.

If $b=0$ then the boundary is a vertical line $z_{1}=-\frac{c}{a}$ and

- if $(a>0$ and $c>0)$ or $(a>0$ and $c<0)$ then the system is controllable in the set $\left\{z \mid z_{2}<0\right\}$ and any trajectory initialized in the set $\left\{z \mid z_{2} \geq 0\right\}$ detaches from $\partial K$,

- if $(a<0$ and $c<0)$ or $(a<0$ and $c>0)$ then the system is controllable in the set $\left\{z \mid z_{2}>0\right\}$ and any trajectory initialized in the set $\left\{z \mid z_{2} \leq 0\right\}$ detaches from $\partial K$.

Let us note that the case $a=b=0$ is meaningless since the system is no longer constrained, hence it is not treated in proposition 1 . The new coordinate frame $\left(x_{1}, x_{2}\right)$ is depicted on figure 2 . We note that the two depicted cases can be rotated to obtain the admissible domain below the boundary. The axis $\left(P, x_{2}\right)$ points inside the admissible set $K$. The dashed arrows on $\partial K$ indicate the directions in which trajectories can be controlled on $\partial K$. Due to the complementarity conditions, it follows that in some regions of $\partial K$, trajectories are restricted to move in a single direction (otherwise they leave $\partial K$ ). The cases when the boundary is vertical, is depicted in figure 3 .

Proof of proposition 1: It is simple to calculate that on $\left\{x \mid x_{2}=0\right\}$ the system has the dynamics

$$
\left\{\begin{array}{c}
\dot{x}_{1}=-\frac{a b}{a^{2}+b^{2}} x_{1}-a u \\
-\frac{a^{2}}{a^{2}+b^{2}} x_{1}+b u \leq 0
\end{array}\right.
$$

If $a \neq 0$ then the feedback $u=-\frac{1}{a}\left(v+\frac{a b}{a^{2}+b^{2}} x_{1}\right)$ yields

$$
\left\{\begin{array}{l}
\dot{x}_{1}=v \\
-x_{1}-\frac{b}{a} v \leq 0
\end{array}\right.
$$

INRIA 
where $v$ is the new input. We notice that if $-x_{1}-\frac{b}{a} v=0$ then the system "grazes" $\partial K$. If $-\frac{a}{b}>0, b \neq 0$, then necessarily $v \leq-\frac{a}{b} x_{1}$, and $v$ can be chosen $<0$ so that $x_{1}$ can be made to decrease while staying on $\partial K$. If $-\frac{a}{b}<0, b \neq 0$, then necessarily $v \geq-\frac{a}{b} x_{1}$. If $x_{1}<0$ then $v>0$, so on $(-\infty, 0), x_{1}$ can only increase. On $(0,+\infty) \ni x_{1}$, one can choose $v=-\frac{a}{b} x_{1}$ so that $P$ is attained only asymptotically from any $x_{1 i}>0$.

Now if $a=0$, the dynamics on $\partial K$ is given by

$$
\left\{\begin{array}{l}
\dot{z}_{1}=-\frac{c}{b} \\
z_{2}=-\frac{c}{b} \\
u+b \lambda=0 \text { and } \lambda \geq 0 \Rightarrow b u \leq 0
\end{array}\right.
$$

The result easily follows. If $b=0$ the dynamics on $\partial K$ is given by

$$
\left\{\begin{array}{l}
z_{1}=-\frac{c}{a} \\
\dot{z}_{2}=u \\
z_{2}+a \lambda=0 \text { and } \lambda \geq 0 \Rightarrow a z_{2} \leq 0
\end{array}\right.
$$

The result is a direct consequence of (7).

Proof of lemma 1: The proof uses the results of proposition 1. To start with, let us consider once again the set $K_{1}$ on figure 1 . Then from proposition 1 it follows that trajectories can only be forced to move from $A^{\prime}$ to $A$, but not the reverse. Consider now $K_{2}$. Then trajectories can be controlled from $E$ to $C$, though $C$ is rechable in infinite time only. Assume that $C$ is just below the axis $\left\{z \mid z_{1}=0\right\}$. It follows from proposition 1 that $\partial K_{2}$ can be tracked clock-wise by applying some suitable switching input. Thus, the points on the right of the vertical line $\left(l^{\prime}\right)$ can be steered to anywhere in $K_{2}$ by first moving on $F E$. One may say that the dynamics is suitably modified on the boundary $F E$ so that $z_{1}$ can decrease in the first quadrant. In the same way the system is $K_{5}-$ controllable and $K_{3}-$ controllable, but it is not $K_{4}$-controllable (the states on the left of the line $(l)$ cannot be reached from anywhere in $K_{2}$ ). The system is $K_{5}-$ controllable since as illustrated a state $z_{f}$ that cannot be attained from $z_{i}$ via a trajectory which remains in $K \backslash \partial K$, can be attained via a path $z_{i} A B z_{f}$.

The proof of lemma 1 is done by observing that under the stated conditions, and from proposition 1 , then any point in $K$ can be steered by $u(\cdot)$ to any other point in $K$. Indeed if a state $z_{f}$ cannot be attained from $z_{i}$ via a trajectory in $K \backslash \partial K$, then a portion of path can be tracked on $\partial K$. For instance from the results of proposition 1, one sees that the boundary of the domain $K_{3}$ on figure 2 can be tracked clockwise. Consequently any point $z_{f}$ on the right of the line $(l)$ can be attained from any point $z_{i}$ on the left of $(l)$. There has to be a portion of the trajectory that evolves on $\partial K$ to reach $z_{f}$ from $z_{i}$. The same reasoning can be done for $K_{5}$. The conditions of lemma 1 are sufficient but can also be seen 
to be necessary, for if one of them fails then there exists couples of states in $K$ which cannot be joined by a controlled trajectory.

We note that in general the input signal $u(\cdot)$ is piecewise continuous.

Remark 3 Let us consider the case $K=\{z \mid g(z) \geq 0\}$ with $g(\cdot)$ a continuous function (possibly differentiable almost everywhere only, i.e. $\partial K$ may have an infinity of corners). It does not suffice to approximate the convex set $K$ by some polyhedron $\bar{K} \subseteq K$, and applying lemma 1 , to conclude about the $\bar{K}$-controllability of the system. Indeed the dynamics is modified on $\partial K$, not on $\partial \bar{K}$. So it is necessary to examine the system on the boundary of $K$ to conclude about controllability.

Remark 4 The systems considered in [14] [15] and in this note are not the same. Discrepancies are: they consider systems that evolve in polytopes $K$ (i.e. bounded convex sets), and the dynamics on the various faces of the polytope are not specified as it is for complementarity systems (which, especially, restricts the choice of inputs on $\partial K$ ). For instance [15, propositions 4.1, 4.2] consider systems with a fixed controlled dynamics $\dot{z}=f(z, u)$, and investigates whether or not there exists a feedback input $u(z)$ such that $z(\cdot)$ can be steered in the polytope. The conditions are essentially on the orientation of the vector field $f(z, u)$ with respect to the polytope faces normal vectors. An interesting field of research would be to link the algorithmic part of [15] and the reachability of complementarity systems, injecting the specificity of complementarity systems in the study.

\section{An example}

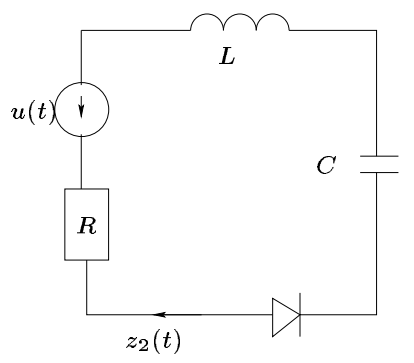

Figure 4: A simple electrical circuit. 
Let us consider the simple electrical circuit in figure 4, where $R$ is a resistor, $L$ is an inductor, $C$ is a capacitor, and the diode is supposed ideal. Its dynamics is given by

$$
\left\{\begin{array}{l}
\dot{z}_{1}=z_{2} \\
\dot{z}_{2}=-\frac{R}{L} z_{2}+\frac{u(t)}{L}-\frac{1}{L C} z_{1}-\frac{1}{L} \lambda \\
0 \leq \lambda \perp-z_{2} \geq 0
\end{array}\right.
$$

where $z_{2}(\cdot)$ is the current across the circuit and $-\lambda$ is the voltage of the diode, $u(\cdot)$ is a voltage control. One sees that this system is not controllable by simple application of lemma 1. One may transform the system in (8) into the canonical form in (1), by applying a pre-feedback $u\left(z_{1}, z_{2}\right)=L v(t)+\frac{R}{L} z_{2}+\frac{1}{L C} z_{1}$. In fact the state $z_{1}(\cdot)$ can only decrease, or be controlled to a constant value on $\partial \Phi$. This is intuitively sound since it corresponds to having the capacitor loaded with a non-positive current at all times.

\section{Conclusion}

In this note we have proposed a characterisation of the controllability properties of planar evolution variational inequalities with control input. These systems are a subclass of complementarity dynamical systems. They are nonsmooth and nonlinear. The material in this note relies heavily on the properties of the system on the boundary of the constraint set and on the behaviour of the trajectories of planar systems in their phase plane. Consequently an extension of this work should rely on the analytical tools in [12] that characterize the control capabilities of a system, on the boundary of its admissible domain. The class of systems that is considered is a narrow class of complementarity dynamical systems. However the results show that the controllabilty of complementarity dynamical systems differs significantly from that of unconstrained systems.

\section{References}

[1] W.P.M.H. Heemels, J.M. Schumacher, S. Weiland, 2000 "Linear complementarity systems", SIAM J. Applied Math., vol.60, no 4, pp.1234-1269.

[2] A. van der Schaft, H. Schumacher, 2000 An Introduction to Hybrid Dynamical Systems, Springer, LNCIS 251, London.

[3] A. Nagurney, D. Zhang, 1996 Projected Dynamical Systems and Variational Inequalities with Applications, Kluwer's International Series in Operations research and Management Science, Boston.

[4] D. Goeleven, B. Brogliato, 2002 "Stability and unstability matrices for linear evolution variational inequalities", submitted.

$\mathrm{RR} \mathrm{n}^{\circ} 4788$ 
[5] D. Zhang, A. Nagurney 1995 "On the stability of projected dynamical systems", J. of Optimization Theory and Applications, vol.85, no 1, pp.97-124.

[6] P. Dupuis, A. Nagurney, 1993 "Dynamical systems and variational inequalities", Annals of Operations Research, vol.44, pp.9-42.

[7] W.P.M.H. Heemels, J.M. Schumacher, S. Weiland, 2000 "Projected dynamical systems in a complementary formalsim", Operations Research Letters, vol.27, pp.83-91.

[8] D. Goeleven, M. Motreanu, V. Motreanu, 2002 "On the stability of stationary solutions of evolution variational inequalities", to appear in Advances in Nonlinear Variational Inequalities.

[9] H.K. Khalil, 1996 Nonlinear Systems, 2nd edition, Prentice-Hall.

[10] M. Kunze, M. Monteiro Marques, 2000 "An introduction to Moreau's sweeping process", in Impacts in Mechanical Systems, Springer Lecture Notes in Physics LNP 551, B. Brogliato (Ed.), pp.1-60.

[11] H. Nijmeijer, A. J. van der Shaft, 1990 Nonlinear Dynamical Control Systems, Springer Verlag.

[12] A.A. ten Dam, E. Dwarshuis, J.C. Willems, 1997 "The contact problem for linear continuous-time dynamical systems: a geometric approach", IEEE Transactions on Automatic Control, vol.42, no 4, pp.458-472, April.

[13] B. Brogliato, 2003 "Some perspectives on the analysis and control of complementarity dynamical systems", to appear in IEEE Transactions on Automatic Control, vol.48, no 6.

[14] L.C.G.J.M. Habets, J.H. van Schuppen, 2001 "A controllability result for piecewiselinear hybrid systems", Proc. of the European Control Conference ECC01, Porto, Portugal (CD Rom).

[15] L.C.G.J.M. Habets, J.H. van Schuppen, 2001 "Control of piecewise-linear hybrid systems on simplices and rectangles", Proc. Workshop Hybrid Systems - Computation and Control, Rome, Italy, 28-30 March; Lecture Notes in Computer Science, Springer Verlag, Berlin, pp.261-274.

[16] A. Bemporad, G. Ferari-Trecate, M. Morari, 2000 "Observability and controllability of piecewise affine and hybrid systems", IEEE Transactions on Automatic Control, vol.45, no 10, pp.1854-1864, October.

\section{Contents}

1 Introduction 
2 Planar evolution variational inequalities

3 Main result

4 An example 10

5 Conclusion

$\mathrm{RR} \mathrm{n}^{\circ} 4788$ 


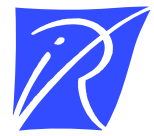

Unité de recherche INRIA Rhône-Alpes

655, avenue de l'Europe - 38330 Montbonnot-St-Martin (France)

Unité de recherche INRIA Futurs : Domaine de Voluceau - Rocquencourt - BP 105 - 78153 Le Chesnay Cedex (France) Unité de recherche INRIA Lorraine : LORIA, Technopôle de Nancy-Brabois - Campus scientifique 615, rue du Jardin Botanique - BP 101 - 54602 Villers-lès-Nancy Cedex (France)

Unité de recherche INRIA Rennes : IRISA, Campus universitaire de Beaulieu - 35042 Rennes Cedex (France) Unité de recherche INRIA Rocquencourt : Domaine de Voluceau - Rocquencourt - BP 105 - 78153 Le Chesnay Cedex (France) Unité de recherche INRIA Sophia Antipolis : 2004, route des Lucioles - BP 93 - 06902 Sophia Antipolis Cedex (France) 